\title{
Pharmaceutical characterization of novel tenofovir liposomal formulations for enhanced oral drug delivery: in vitro pharmaceutics and Caco-2 permeability investigations
}

\author{
This article was published in the following Dove Press journal: \\ Clinical Pharmacology:Advances and Applications \\ 23 February 2017 \\ Number of times this article has been viewed
}

\author{
Crystal B Spinks' \\ Ahmed S Zidan ${ }^{2,3}$ \\ Mansoor A Khan ${ }^{4}$ \\ Muhammad J Habib' \\ Patrick J Faustino ${ }^{2}$ \\ 'Department of Pharmaceutical \\ Sciences, School of Pharmacy, Howard \\ University, Washington, DC, ${ }^{2}$ Division \\ of Product Quality Research, Office \\ of Pharmaceutical Quality, Food and \\ Drug Administration, Silver Spring, \\ MD, USA; ${ }^{3}$ Faculty of Pharmacy, \\ Zagazig University, Zagazig, Egypt; \\ ${ }^{4}$ Irma Lerma Rangel College of \\ Pharmacy, Texas A\&M Health Science \\ Center, College Station, TX, USA
}

\begin{abstract}
Tenofovir, currently marketed as the prodrug tenofovir disoproxil fumarate, is used clinically to treat patients with HIV/AIDS. The oral bioavailability of tenofovir is relatively low, limiting its clinical effectiveness. Encapsulation of tenofovir within modified long-circulating liposomes would deliver this hydrophilic anti-HIV drug to the reticuloendothelial system for better therapeutic efficacy. The objectives of the current study were to prepare and pharmaceutically characterize model liposomal tenofovir formulations in an attempt to improve their bioavailability. The entrapment process was performed using film hydration method, and the formulations were characterized in terms of encapsulation efficiency and Caco-2 permeability. An efficient reverse-phase high-performance liquid chromatography method was developed and validated for tenofovir quantitation in both in vitro liposomal formulations and Caco-2 permeability samples. Separation was achieved isocratically on a Waters Symmetry C8 column using $10 \mathrm{mM} \mathrm{Na}_{2} \mathrm{PO}_{4} /$ acetonitrile $\mathrm{pH} 7.4(95: 5 \mathrm{v} / \mathrm{v})$. The flow rate was $1 \mathrm{~mL} / \mathrm{min}$ with a $12 \mathrm{~min}$ elution time. Injection volume was $10 \mu \mathrm{L}$ with ultraviolet detection at $270 \mathrm{~nm}$. The method was validated according to United States Pharmacopeial Convention category I requirements. The obtained result showed that tenofovir encapsulation within the prepared liposomes was dependent on the employed amount of the positive charge-imparting agent. The obtained results indicated that calibration curves were linear with $r^{2}>0.9995$ over the analytical range of $1-10 \mu \mathrm{g} / \mathrm{mL}$. Inter- and intraday accuracy and precision values ranged from $95 \%$ to $101 \%$ and $0.3 \%$ to $2.6 \%$, respectively. The method was determined to be specific and robust. Regarding the potential of the prepared vectors to potentiate tenofovir permeability through the Caco-2 model, a 10-fold increase in tenofovir apparent permeability was observed compared to its oral solution. In conclusion, this novel and validated method was successfully applied to characterize both in vitro encapsulation efficiency and Caco-2 permeability transport for the pharmaceutical assessment of novel tenofovir formulations.
\end{abstract}

Keywords: liposomes, tenofovir, targeting, chromatography, entrapment, permeation

\section{Introduction}

Tenofovir (9-[-(R)-2(phosphonomethoxy)propyl]adenine) (Figure 1A), known as PMPA, is the base form of the prodrug tenofovir disoproxil fumarate (TDF; Figure 1B). Recently, it is also proposed as another prodrug tenofovir alafenamide (TAF; formerly known as GS-7340) with improved properties relative to TDF. Compared to TDF, TAF-containing regimens were associated with a $90 \%$ reduction in plasma tenofovir concentrations and improved renal and bone safety. ${ }^{1}$ TDF therapy has been associated
Correspondence: Patrick J Faustino Division of Product Quality Research, and Drug Administration, Life Science Building 64, 10903 New Hampshire Avenue, Silver Spring, MD 20993, USA Tel +l 30I 7960021

Fax + I 3017969816

Email patrick.faustino@fda.hhs.gov 
A

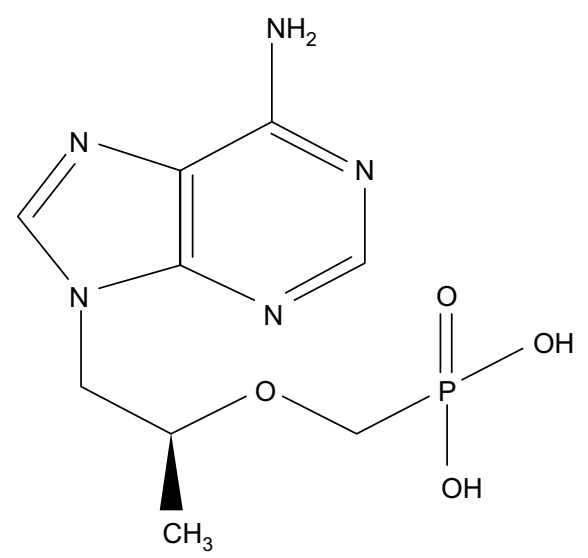

B

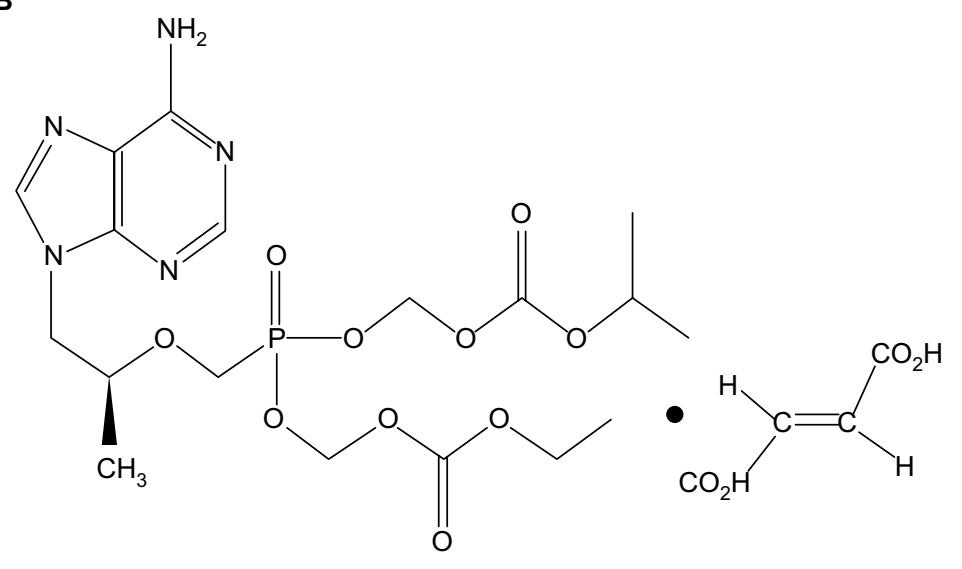

Figure I Chemical structure of $(\mathbf{A})$ tenofovir and $(\mathbf{B})$ tenofovir disoproxil fumarate.

with changes in markers of renal function, decreases in bone mineral density, and a rare occurrence of serious renal adverse events, including Fanconi's syndrome. ${ }^{2}$ TDF is indicated in combination with other antiretroviral drugs for the treatment of adult patients infected with HIV, ${ }^{3}$ who are intolerant to nucleoside analog therapy. ${ }^{4-6}$ To achieve its therapeutic efficacy, mechanistically, TDF requires intracellular activation through phosphorylation..$^{7-9}$ Phosphorylation occurs by cellular enzymes to form TDF diphosphate, the active form of the drug. The recommended dosage regimen of TDF is presented as once daily due primarily to its long biological half-life. ${ }^{8}$ Tenofovir is hydrophilic and demonstrates low oral bioavailability in animal studies. ${ }^{4}$ It is a white crystalline substance developed originally as an acyclic phosphonate nucleotide analog. ${ }^{3}$ It has a solubility of $\sim 5 \mathrm{mg} / \mathrm{mL}$ in aqueous medium, while the solubility of the prodrug TDF is $\sim 2.5$-fold higher at $13.4 \mathrm{mg} / \mathrm{mL} .^{5}$ The improvement of aqueous solubility and the oral dosing with food have been shown to further enhance the bioavailability of TDF..$^{10}$ Despite these factors, the bioavailability of TDF is still relatively low (ie, $25 \%-30 \%$ ). ${ }^{8}$ Therefore, it has been suggested that other drug delivery strategies such as liposomes could enhance the bioavailability of tenofovir or TDF. ${ }^{7,11}$ Liposomal encapsulation offers two advantages: first, the effective delivery of the hydrophilic molecule across the gastrointestinal epithelial wall, and second, the protection for the prodrug to optimize therapeutic efficacy.

According to its chemical nature, TDF molecules exhibit negative charges that facilitate its wide systemic distribution and disposition to different tissues for extended periods. ${ }^{12}$ As a result, TDF might demonstrate low selectivity of kinases on its activation ${ }^{13}$ and therapeutic variability among patients. ${ }^{14}$ On the other hand, administration of the tenofovir base would eliminate this step because it is already monophosphorylated; hence, more consistent therapeutic efficacy would be anticipated. Therefore, drug delivery strategies for tenofovir base are deemed important to overcome the relatively low bioavailability encountered in this case. ${ }^{15}$ Liposomal drug delivery strategies are one of the most important approaches to improve the cellular uptake and subsequent bioavailability of drugs such as tenofovir. ${ }^{16}$ Following administration, liposomes are recognized and taken up by cells of the mononuclear phagocytic system. Since HIV localizes in these cells, liposomes therefore represent an optimal drug delivery system for targeting antiretroviral agents into infected cells and thus have the potential of improving the efficacy of antiretroviral drugs and reducing the side effects. ${ }^{7,17,18}$ Consequently, a liposomal formulation of tenofovir would offer advantages of both improving its bioavailability and facilitating its targeting to the mononuclear phagocytic system.

The implementation of new formulation approaches is part of the Agency's critical path initiative to facilitate the introduction of new medical products. Liposomal formulation for HIV/ AIDS prodrugs, and the ability to fully characterize them, is an area that has not been fully explored. FDA manufacturing and pharmaceutical characterization facilities provide the scientific opportunity to develop or implement innovative pharmaceutical characterization technologies for complex drug delivery systems. The novel application of pharmaceutical characterization technologies, such as high-performance liquid chromatography (HPLC) and in vitro drug permeability for liposomal HIV/AIDS products, will enhance regulatory understanding for science-based decisions.

In order to characterize the feasibility of these model liposomal HIV/AIDS formulations, it is necessary to analyze tenofovir encapsulation efficiency in its liposomal formulations. ${ }^{19}$ Therefore, there is a need for a robust validated HPLC method that will accurately determine the amount of tenofovir 
encapsulated in the liposomal matrix. Currently, there is no simple, efficient, yet validated reverse-phase isocratic method available for the pharmaceutical assessment of in vitro tenofovir samples. However, different approaches have been proposed in the literature to analyze TDF. ${ }^{4,20}$ Bioanalytical samples of tenofovir are very commonly analyzed with a variety of chromatographic methods such as HPLC, ${ }^{21,22}$ electrospray ionization triple quadrupole-mass spectrometry, ${ }^{23}$ tandem LC-MS mass spectrometry, ${ }^{24}$ and capillary depletion analysis. ${ }^{25}$ A solid-liquid extraction technique was coupled with chromatographic analysis for the determination of TDF. ${ }^{4}$ Rezk et $\mathrm{al}^{26}$ have also described the concomitant determination of TDF and emtricitabine in human plasma using HPLC after solid-phase extraction. These proposed analytical methods were limited by their high limit of detection (LOD) and limit of quantitation (LOQ). Having these limitations, the development of a simple yet reliable UV detection-based reversephase HPLC (RP-HPLC) method to quantify tenofovir in both liposomal formulations and Caco- 2 media was necessary. The published analytical methods for the analysis of tenofovir for the assessment of liposomal formulations are scarce. This is a result of the lipophilic sample matrix that irreversibly interacts with HPLC resins and negatively affects the chromatography and method robustness. Consequently, the objectives of the characterization part of the current study were to develop and validate a novel, simple, and efficient method for the accurate analysis of complex-matrix tenofovir in vitro samples. Moreover, an attempt to test this method was applied for the pharmaceutical assessment of both the liposomal entrapment efficiency (EF) and the in vitro liposomal formulation permeability using a Caco-2 in vitro cell model.

\section{Experimental Materials}

Tenofovir was purchased from Hangzhou Starshine Pharmaceutical Company (Hangzhou, China). Dideoxy-cytosine (ddC) and HPLC-grade dibasic sodium phosphate were purchased from Sigma-Aldrich (St Louis, MO, USA). Hydrogenated phosphatidylcholine ( $95 \%$ hydrogenated phosphatidylcholine and $0.5 \%$ hydrogenated lysophosphatidylcholine) was supplied from American Lecithin (Oxford, CT, USA). Cholesterol, stearylamine, phosphoric acid, and HPLC-grade acetonitrile were purchased from VWR (West Chester, PA, USA). Phosphate buffer solution $\mathrm{pH}$ 6.8, sodium chloride, sucrose, sodium phosphate, and chloroform were purchased from Fisher Scientific (Pittsburgh, PA, USA). Caco-2 cell line was obtained from American Type Culture Collection (Manassas, VA, USA). Cell culture media and reagents were purchased from Invitrogen Inc. (Carlsbad, CA, USA) and Life
Technologies, Inc. (Grand Island, NY, USA). HPLC-ready $18 \mathrm{MW}$ water (deionized [DI] water) was obtained, in-house, from a Milli-Q Gradient A-10 water purification system, Millipore Corporation (Bedford, MA, USA).

\section{Instrumentation and chromatographic conditions}

The HPLC system consisted of a Hewlett Packard 1050 series (Agilent Technologies, Wilmington, DE, USA) equipped with a quaternary pump, online degasser, column heater, autosampler, and diode array detector (DAD). Data collection and analysis were performed using ChemStation software (Agilent Technologies). Separation was achieved on a Water's symmetry C8 column $\left(250 \times 4.6 \mathrm{~mm}^{2}\right), 5 \mathrm{~mm}$ particle size (Waters Corporation, Milford, MA, USA). The elution was isocratic with a mobile-phase composition of acetonitrile: $10 \mathrm{mM}$ phosphate buffer solution ( $\mathrm{pH} 7.4 ; 5: 95 \mathrm{v} / \mathrm{v})$. The column temperature was maintained at $26^{\circ} \mathrm{C}$. The injection volume was $10 \mu \mathrm{L}$ with UV detection set at $270 \mathrm{~nm}$.

\section{Preparation of standard solutions}

To prepare the calibration samples, stock solution I of $1 \mathrm{mg} / \mathrm{mL}$ of tenofovir was prepared using tenofovir active pharmaceutical ingredient (API) in DI water. The working standard was further diluted to $0.1 \mathrm{mg} / \mathrm{mL}$. The standard calibration curve was formed from six levels prepared by making six serial dilutions from stock solution I with HPLC mobile phase to final concentrations of $1,2,4,6,8$, and $10 \mathrm{mg} / \mathrm{mL}$ for an analytical range from 1 to $10 \mathrm{mg} / \mathrm{mL}$. To prepare quality control (QC) samples, stock solution II of $1 \mathrm{mg} / \mathrm{mL}$ for tenofovir was prepared using tenofovir API in DI water. The working standard was prepared in DI water and further diluted to $0.1 \mathrm{mg} / \mathrm{mL}$. QC samples at the low, intermediate, and high QC levels of the analytical range were diluted with mobile phase to a final concentration of 1 , 6 , and $10 \mathrm{mg} / \mathrm{mL}$, respectively. Stock solution of $0.5 \mathrm{mg} / \mathrm{mL}$ for $\mathrm{ddC}$ was prepared in mobile-phase solvent. The working standard was diluted to $5 \mu \mathrm{g} / \mathrm{mL}$. Samples were diluted with mobile phase to a final concentration of $2.5 \mathrm{mg} / \mathrm{mL}$

\section{System suitability}

The system suitability standard solution containing $2 \mathrm{mg} / \mathrm{mL}$ tenofovir and a resolution standard of $2 \mu \mathrm{g} / \mathrm{mL} \mathrm{ddC}$ was prepared by diluting tenofovir and $\mathrm{ddC}$ in mobile phase at 1:50 and 1:5 dilutions, respectively, from the stock solution. System suitability was determined by making six replicate injections of the standard. The acceptance criteria were $<2 \%$ relative standard deviation ( $\%$ RSD) for peak area, $>3000$ for column plates, $<0.25$ min for peak width, $>3$ for resolution factor, and $<1.25$ for United States Pharmacopeial Convention 
(USP) tailing factor. These results were used to evaluate critical operating parameters on the chromatographic system, hence assuring system acceptability each time the method was used.

\section{Method validation and robustness}

The method was validated according to the United States Pharmacopeia category I requirements for assay. The validation characteristics addressed were linearity, range, accuracy, precision, specificity, and robustness. Specificity was evaluated by demonstrating that no peaks in the chromatogram of the blank sample that were consistent with the retention time of tenofovir and observing no coeluting peaks in the tenofovir sample. Additionally, the Agilent ChemStation peak purity software was used to evaluate diode array spectral data of the chromatographic peak to determine whether impurities were present or coeluting with tenofovir. Accuracy and precision were determined by analyzing QC standard samples at three concentrations of tenofovir $(1,6$, and $10 \mathrm{mg} / \mathrm{mL})$ over the analytical range. The method precision was determined by injecting five standard QC samples once at each concentration level for the intraday precision and on 3 days for the intermediate precision. Precision was expressed by \%RSD of the analyte peaks. Accuracy was established by evaluating the amount determined from the quality control standards and comparing to the respective nominal value expressed as percent recovery. The LOD was defined as the concentration that yields a signal-to-noise ratio of $3: 1$. On the other hand, the lowest concentration levels that could be determined with a percentage of deviation from the nominal concentration and relative standard deviation $<20 \%$ was considered the lowest LOQ.

Standard calibration curves were prepared with six calibrators over a concentration range of $1-10 \mathrm{mg} / \mathrm{mL}$ for tenofovir and $5 \mathrm{mg} / \mathrm{mL}$ for ddC. The chromatographic graph of peak area versus the drug concentrations was treated by linear least square regression analysis. The standard curves were evaluated for interday reproducibility. The linear regression of the standard curve met acceptance criteria at $R^{2}>0.9995$. The analytical range was established by demonstrating acceptable accuracy, precision, and linearity over the analytical range. The robustness of the method was evaluated by analyzing the system suitability standard $(n=6)$ through system variations in elution parameters. The parameters were varied individually by adjusting the HPLC pump flow rate $( \pm 2.5 \%)$, column temperature $\left( \pm 2{ }^{\circ} \mathrm{C}\right)$, autosampler injector volume $( \pm 25 \%)$, mobile-phase acetonitrile composition $( \pm 2 \%)$, and mobile-phase $\mathrm{pH}$ ( \pm 0.1 units).

\section{Liposome preparation and EF}

Multilamellar liposomes incorporating tenofovir were prepared by the thin film method as described by Zidan et al. ${ }^{27}$
Briefly, two liposomal formulations were prepared using $50 \mathrm{mg}$ cholesterol, either $7.5 \%$ (formulation $\mathrm{A}$ ) or $15 \%$ (formulation B) stearylamine as a positive charge imparting agent and an amount of phospholipon $100 \mathrm{H}$ to make a total lipid pool of $150 \mathrm{mg}$. In a round-bottomed flask, cholesterol and the specified amounts of phospholipon and stearylamine were dissolved in $10 \mathrm{~mL}$ of chloroform. The organic solvent was removed under reduced pressure using a rotary evaporator (RotavaporÒ R-210/215; BÜCHI Labortechnik AG, Postfach, Switzerland, USA), at $63^{\circ} \mathrm{C}$, to deposit a thin film of dry lipid on the walls of the flask. Evaporation was continued for 15 min after the dry residue appeared. The film was then purged with nitrogen for $5 \mathrm{~min}$ followed by overnight vacuum drying at room temperature for complete solvent evaporation. To each flask, $5 \mathrm{~mL}$ of isotonic tenofovir solution $(5 \mathrm{mg} / \mathrm{mL})$ was added. The flask was attached to a rotary evaporator and rotated at $63^{\circ} \mathrm{C}$ for $30 \mathrm{~min}$. The liposomes, which were formed, were harvested by ultracentrifugation (Eppendorf Centrifuge, Model 5415 C; Eppendorf-Netheler-Hinz GmbH 2000, Hamburg, Germany) at 65,000 rpm for $2 \mathrm{~h}$. The resulting supernatant was used for indirect estimation of the EF.

Tenofovir EF was determined by measuring the total amount of the drug loaded in liposomal samples (ie, experimental loading) and comparing this value with the expected amount of the drug in each of the samples based on the drug loading during the preparation (ie, theoretical loading). The EF was calculated using the following equation, where Dt is the total amount of tenofovir used in the hydration medium and $\mathrm{Dm}=\mathrm{Dt}$ - amount of free drug in supernatant:

$$
\mathrm{EF}=\frac{\mathrm{Dm} \times 100}{\mathrm{Dt}}
$$

\section{Permeability assessment}

Caco-2 permeability study is used to assess the permeability of tenofovir liposomal formulations. Caco-2 human epithelial cells (Caucasian colon adenocarcinoma; purchased from Sigma-Aldrich Corporation, St. Louis, MO, USA) from a passage number between 30 and 50 were used to evaluate the permeability of tenofovir liposomal formulations. ${ }^{28}$ Caco-2 cells were maintained in Dulbecco's Modified Eagle's Medium with high glucose $(4.5 \mathrm{~g} / \mathrm{L})$ that was supplemented with $10 \%$ fetal bovine serum, $1 \%$ nonessential amino acids, $1 \%$ penicillin streptomycin solution, and $2 \%$ glutamine. The cells were seeded at a cell density of $\sim 10^{5}$ cells $/ \mathrm{cm}^{2}$ on transwell-clear polyester membrane filter inserts $(0.45 \mu \mathrm{m}$ pore size, $1.13 \mathrm{~cm}^{2}$; Corning Costar, Cambridge, MA, USA) in a 12-well transwell plate format. The apical (AP) and 
basolateral (BA) compartments received 0.5 and $1.5 \mathrm{~mL}$ of the culture media. Four transwells were used for the API or the liposomal formulations, respectively. The monolayer integrity was assessed by inverted light microscopy and by the measurement of transepithelial electrical resistance (TEER) using a MillicellÒ-ERS apparatus (Millipore Corporation). Caco-2 monolayers exhibiting a TEER value of $>250 \Omega \mathrm{cm}^{2}$ were used within 30 days postseeding.

For permeability experiments, the culture media were removed from both AP and BA sides, and the monolayers were washed twice with cold phosphate-buffered saline with calcium and magnesium. The monolayers were preincubated at $37^{\circ} \mathrm{C}$ for $30 \mathrm{~min}$ in a $\mathrm{CO}_{2}$ incubator with prewarmed transport media (TM). The TM consisted of Hank's balanced salt solution with calcium and magnesium with the AP ( $\mathrm{pH}$ 6.8) and BA ( $\mathrm{pH}$ 7.4) buffers supplemented with $10 \mathrm{mM}$ 2-( $N$-morpholino) ethane-sulfonic acid and $25 \mathrm{mM}$ $N$-(2-hydroxyethyl) piperazine- $N \phi$-2-ethane-sulfonic acid, respectively. The $\mathrm{pH}$ of the transport buffers was adjusted with either sodium hydroxide or hydrochloric acid. At the end of the preincubation period, the TM was removed, and the appropriate volume of either the control tenofovir solution or the reconstituted liposomal formulation in TM was added to the AP side. The liposomal residues were reconstituted with the TM for final drug concentrations based on the encapsulation efficiency data. The control tenofovir drug solutions were prepared with Hank's balance salt solution at 10 to 20 times the desired final concentrations. Approximately $10 \mu \mathrm{L}$ of AP samples $\left(\mathrm{C}_{0}\right)$ were taken immediately after the addition of compound to the AP side (zero time) and at the end of experiments (120 min) for HPLC analysis. Approximately $10 \mu \mathrm{L}$ of samples were taken from the BA side at 30, 60, 90, and $120 \mathrm{~min}$ for HPLC analysis to assess permeability. These time points were determined to be appropriate since this sampling scheme provided a similar estimate of the permeability compared to that from preliminary studies with a more extensive number of time points. The removed BA sample volumes were replenished with equal volumes of the fresh TM for the BA side. The TEER was measured both in the beginning and at the end of experiment. Samples were diluted as required in mobile phase and injected onto the HPLC.

The apparent permeability $\left(\mathrm{P}_{\text {app }} \times 10^{-6} \mathrm{~cm} / \mathrm{s}\right)$ was calculated using the following equation:

$$
P_{\text {app }}=\frac{V_{R}}{A \times C_{0}} \times \frac{d c}{d t}
$$

where $V_{R}$ is the volume of the receiving chamber, $A$ is the monolayer filter surface area $\left(1.13 \mathrm{~cm}^{2}\right), \mathrm{C}_{0}$ is the initial drug concentration in $\mathrm{mg} / \mathrm{mL}$ in the donor chamber, and $\mathrm{dc} /$ $\mathrm{dt}$ is the initial slope of the concentration versus time curve. ${ }^{29}$

\section{Results and discussion Development of HPLC method}

Tenofovir is a hydrophilic molecule $(\log \mathrm{P} 1.3)$ with a $\mathrm{pKa}$ of 3.75 that is poorly retained on most RP-HPLC columns. Therefore, there was a need to optimize an HPLC method that would ensure selectivity, sensitivity, and an acceptable capacity factor. The goal was to develop and validate an HPLC isocratic method that would be suitable for the characterization of tenofovir liposomal formulations 1) with acceptable selectivity and an efficient analysis time and 2) without interference from liposomal or Caco-2 matrix effects. Method development was focused on evaluating column type, buffer type and strength, $\mathrm{pH}$ of the mobile phase, and type of organic solvent, while making small sequential adjustments to optimize the chromatographic selectivity and resolution. The initial method development specifically focused on exploiting the potential selectivity of tenofovir based on its aromatic structure and alkyl side chain with a $\mathrm{C}-18$ sorbent. However, although tenofovir did not coelute with the solvent front, isocratic elution at organic compositions of 5\%-10\% resulted in poor peak symmetry represented by severe fronting of the chromatographic peak and subsequent carry over of trace amounts of tenofovir. Paradoxically, although tenofovir is very polar, trace amounts $(<1 \%)$ were strongly retained to C-18 column after several injections. Several column wash procedures were used including a buffer washout and a complimentary 0:90\% v/v acetonitrile wash, but they were insufficient to correct the problem for long-term $\mathrm{C}$ - 18 column use. Analytical range and sensitivity were also negatively impacted by the trace levels of tenofovir that were strongly bound to the $\mathrm{C}-18$ column. Extending the analytical range to higher concentration levels resulted in the appearance of trace levels of tenofovir more quickly following wash procedures. Because tenofovir has a relatively low molar absorptivity, ${ }^{30}$ these chromatographic issues prevented the accurate analysis of tenofovir at low concentrations and required a column change from $\mathrm{C} 18$ to $\mathrm{C} 8$ to reduce the column retention of trace amounts of drug and enhance peak symmetry.

Adjusting the $\mathrm{pH}$ of the mobile-phase aqueous component improved peak resolution and retention time on the $\mathrm{C}-8$ column. A buffer $\mathrm{pH}$ of 2.7 gave a retention time of $3.64 \mathrm{~min}$ and resolution of 1.85 with ddC, whereas the retention time and resolution of $4.5 \mathrm{~min}$ and 14.63 , respectively, were established when the $\mathrm{pH}$ was adjusted to 7.4. Adjusting the organic solvent percentage was important to prevent the coelution of tenofovir with ddC, while maintaining an acceptable capacity 
factor. The organic modifier, acetonitrile, varied between $3 \%$ and $7 \%$, where the higher concentration resulted in a more efficient retention time. Increasing the organic modifier shifted the retention time closer to the solvent front and vice versa with decreased organic modifier. The composition of the organic modifier was adjusted to $5 \%$ producing an optimal retention time of $\sim 4.1 \mathrm{~min}$. With the adjustment of these chromatographic variables, the method was able to achieve an acceptable k', peak symmetry, and a resolution factor (Table 1) of the system suitability standard.

\section{System suitability and method validation}

The system suitability test verifies that the resolution and reproducibility of the chromatographic system are adequate for the analysis to be conducted. ${ }^{31}$ All critical parameters tested ( $\mathrm{k}^{\prime}$, peak area RSD, retention time RSD, column plates, tailing, etc.) met the acceptance criterion on all days (Table 1). Adequate resolution of $>3$ between the tenofovir and the ddC peak ensured the specificity of the method to analyze both compounds. The following method validation characteristics were addressed for tenofovir: accuracy, precision, specificity, LOQ, linearity, range, and robustness. The method was found to be acceptable according to the requirements for USP category I. Specificity was established by observing no peaks in the blank chromatogram and no coeluting peaks in the tenofovir sample as shown in Figure 2. Additionally, the Agilent ChemStation peak purity software was used to evaluate diode array spectral data of the chromatographic peak and determined that no coeluting impurities were present with tenofovir.

Linearity of the method was established by preparing standard calibration curves over the analytical range of $1-10 \mathrm{mg} / \mathrm{mL}$ for tenofovir. This narrow but chromatographically necessary analytical range was first a result of tenofovir sensitivity where at $1 \mathrm{mg} / \mathrm{mL}$ sensitivity was lost. Moreover, working concentrations $>10 \mathrm{mg} / \mathrm{mL}$ resulted in a significant quantity of tenofovir remaining on the column; hence, excessive washing with mobile phase was required. The results in Table 2

Table I System suitability data of six injections of $2 \mu \mathrm{g} / \mathrm{mL}$ tenofovir and $2 \mu \mathrm{g} / \mathrm{mL} \mathrm{ddC}$ as a resolution standard

\begin{tabular}{llllll}
\hline USP criteria & Specification & Day I & Day 2 & Day 3 & Pass/fail \\
\hline Retention time & $\leq 2.0 \%$ & $0.248 \%$ & $0.249 \%$ & $0.085 \%$ & Pass \\
Capacity factor k' & $>0.3$ & 0.623 & 0.670 & 0.698 & Pass \\
Symmetry & $>0.50$ & 0.612 & 0.619 & 0.630 & Pass \\
Area & $\leq 2.0 \%$ & $0.328 \%$ & $0.288 \%$ & $0.237 \%$ & Pass \\
Theoretical plates & $>3000$ & 22,361 & 22,402 & 22,027 & Pass \\
USP tailing & $>1.75$ & 1.609 & 1.587 & 1.557 & Pass \\
Resolution & $>3.0$ & 15.119 & 15.024 & 14.895 & Pass \\
\hline
\end{tabular}

Abbreviations: ddC, dideoxy-cytosine; USP, United States Pharmacopeial Convention.

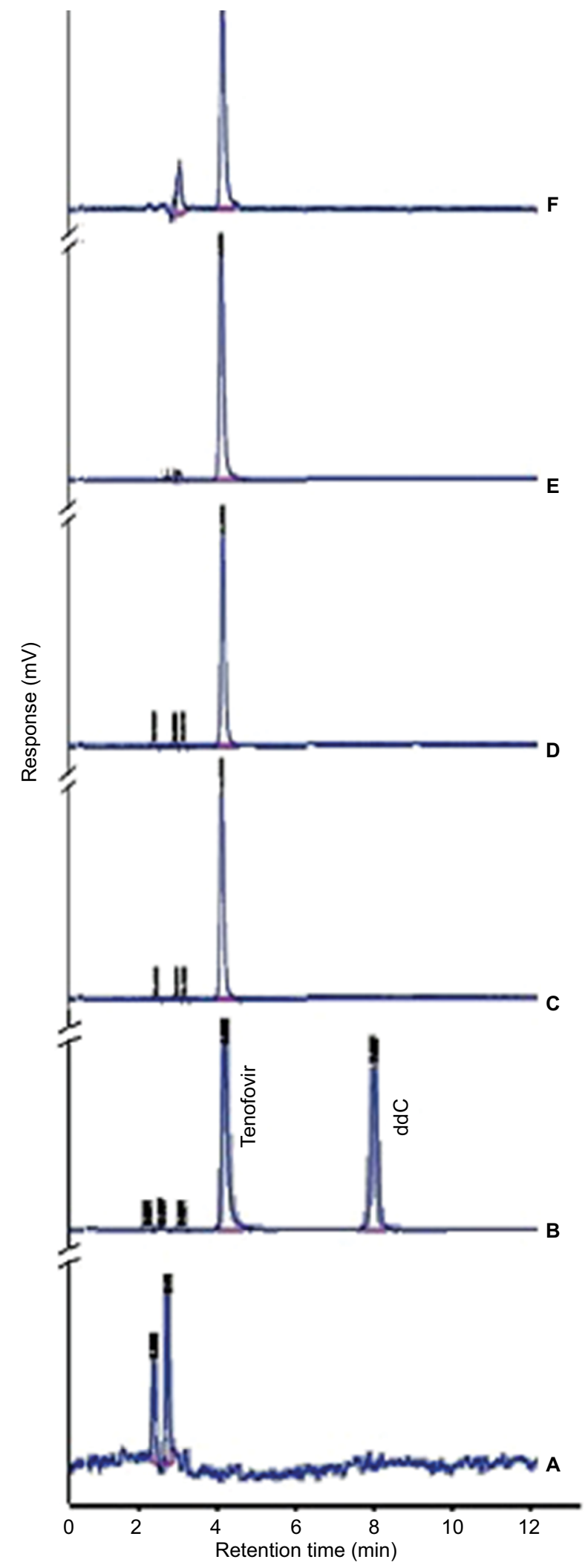

Figure 2 HPLC chromatograms for (A) blank, (B) system suitability $(6 \mu \mathrm{g} / \mathrm{mL}$ tenofovir and $6 \mu \mathrm{g} / \mathrm{mL} \mathrm{ddC)}$, (C) EF sample of formulation A, (D) EF sample of formulation B, (E) Caco-2 study sample of formulation A, and (F) Caco-2 study sample of formulation B. Formulations were prepared using $50 \mathrm{mg}$ cholesterol, either $7.5 \%$ (formulation A) or 15\% (formulation B) stearylamine as a positive charge imparting agent and an amount of phospholipon $100 \mathrm{H}$ to make a total lipid pool of $150 \mathrm{mg}$. Abbreviations: HPLC, high-performance liquid chromatography; EF, entrapment efficiency; ddC, dideoxy-cytosine. 
Table 2 Linearity, accuracy, and precision data of tenofovir analysis

\begin{tabular}{|c|c|c|c|c|c|}
\hline \multicolumn{6}{|c|}{ Linearity data of tenofovir calibration sets } \\
\hline \multirow{2}{*}{$\begin{array}{l}\text { Standard } \\
\text { curve } \\
\text { Validation I }\end{array}$} & \multirow{2}{*}{$\begin{array}{l}\begin{array}{l}\text { Range } \\
(\mu \mathrm{g} / \mathrm{mL})\end{array} \\
1.0-10\end{array}$} & Calibrators & \multicolumn{2}{|c|}{ Slope $y$-Intercept } & $R^{2}$ value \\
\hline & & 6 & 0.0566 & $5-0.0691$ & 0.9995 \\
\hline Validation 2 & $1.0-10$ & 6 & 0.0579 & -0.0948 & 0.9996 \\
\hline Validation 3 & $1.0-10$ & 6 & 0.0603 & $3-0.0166$ & 0.9995 \\
\hline \multicolumn{6}{|c|}{ Accuracy and precision: drug substance $(n=6)$} \\
\hline $\begin{array}{l}\text { Validation } \\
\text { parameters }\end{array}$ & $\begin{array}{l}\text { Validation } \\
\text { solutions }\end{array}$ & $\mathrm{I} \mu \mathrm{g} / \mathrm{mL}$ & $\begin{array}{l}2 \mu \mathrm{gg} / \\
\mathrm{mL}\end{array}$ & $6 \mu \mathrm{g} / \mathrm{mL}$ & $\begin{array}{l}10 \mu \mathrm{g} / \\
\mathrm{mL}\end{array}$ \\
\hline \multirow[t]{3}{*}{ Accuracy (\%) } & Set I & 94.6 & 96.8 & 101.2 & 99.4 \\
\hline & Set 2 & 95.5 & 98.6 & 100.0 & 99.5 \\
\hline & Set 3 & 98.0 & 99.1 & 100.7 & 99.8 \\
\hline Precision & Set I & 2.58 & 1.51 & 0.625 & 1.15 \\
\hline \multirow[t]{2}{*}{ (\%RSD) } & Set 2 & 0.84 & 1.17 & 0.334 & 0.49 \\
\hline & Set 3 & 0.98 & 1.61 & 0.72 & 1.70 \\
\hline
\end{tabular}

Abbreviation: RSD, relative standard deviation.

show a linear correlation between analyte peak area and the concentration of the drug over the analytical range with $r^{2} \geq$ 0.999. Accuracy and precision were established across the analytical range for tenofovir. The intra- and interday accuracy and precision were calculated using multiple injections of the QC samples. Results for the intraday accuracy and precision of tenofovir are summarized in Table 2 . The accuracy values ranged from $94.6 \%$ to $101.2 \%$ for all QC samples. The precision of the analytical method was evidenced by the low $\%$ RSD that did not exceed $2.6 \%$.

According to the determined signal-to-noise ratio, tenofovir presented LOD of $0.21 \mu \mathrm{g} / \mathrm{mL}$ and LOQ of $0.37 \mu \mathrm{g} / \mathrm{mL}$. As the objective of the method was the quantitation of tenofovir in both in vitro pharmaceutical solutions and media of Caco- 2 cells culture, these obtained values of LOD and LOQ should be considered as the limit of method sensitivity. Hence, the developed method was more sensitive than other reported analytical methods in the literature. For example, Ramaswamy and Arul Gnana Dhas ${ }^{32}$ reported LOD and LOQ values of $0.38,0.76$, and $0.94 \mu \mathrm{g} / \mathrm{mL}$ and $1.25,2.5$, and $3.10 \mu \mathrm{g} / \mathrm{mL}$ for pharmaceutical samples of emtricitabine, tenofovir, and efavirenz, respectively. These reported values were obtained using a Zorbax SB CN $(250 \times 4.6 \mathrm{~mm}, 5 \mu \mathrm{m})$ column at $260 \mathrm{~nm}$ UV detection. On another study, Ashour and Belal ${ }^{33}$ has reported LOD of $0.6009 \mu \mathrm{g} / \mathrm{mL}$ and LOQ of $1.8210 \mu \mathrm{g} / \mathrm{mL}$ for the detection of tenofovir in its binary solutions with emtricitabine at 228.5 and $260.5 \mathrm{~nm}$, respectively. Their reported method was successful in determination of concentrations of tenofovir and emtricitabine in their analytical samples of quality assessment of solid dosage forms.

Robustness is a measure of the method's capability to remain unaffected by small deliberate changes in method parameters. ${ }^{34}$ The robustness of the method was evaluated by making small adjustments to the operating parameters of the system suitability procedure. Table 3 shows data of system suitability parameters at low and high values of these intended changes for multiple injections. The obtained data show that the method could accept small deliberate changes to the operating conditions while maintaining its accuracy, precision, and reproducibility. All robustness data remained $<5 \% \mathrm{RSD}$ for each adjusted parameter with the exception of increased $\mathrm{pH}$ from nominal to high. For example, the retention time changed from $4.055 \pm 0.092$ to $3.89 \pm 0.069 \mathrm{~min}$ by changing the organic composition of the mobile phase from $5 \%$ to $5.1 \%$, respectively. On the other hand, the capacity factor $\left(\mathrm{k}^{\prime}\right)$ changed from $0.674 \pm 0.225$ to $0.595 \pm 0.253$ by varying the temperature of the column from $26^{\circ} \mathrm{C}$ to $28^{\circ} \mathrm{C}$, respectively. Variability increased to $6.1 \%$ RSD due to method sensitivity to increase in $\mathrm{pH}$ value. As a result, none of the adjustments caused a significant change $(p<0.05)$ in resolution between tenofovir system suitability standard and ddC, peak area, \%RSD, peak width, theoretical plates, and/or USP tailing factor.

\section{Analysis of the tenofovir liposomal formulation}

The validated method was used as a pharmaceutics tool to determine the efficiency and capacity of the liposomal formulation to entrap tenofovir. It was also used as a biopharmaceutics tool to analyze samples from the Caco-2 permeability study and assess the potential for oral bioavailability enhancement. Indirect determination of the EF was done by collecting the supernatant of each formulation, following the centrifugation cycle. Each sample was diluted with mobile phase and injected onto the HPLC using the described validated method. Representative chromatograms of supernatant analysis for tenofovir liposomal formulations A and B are shown in Figure 2. The chromatograms showed that there was no interference from the liposomal components with the drug peaks. Table 4 shows the capacity of the proposed liposomal formulations to entrap tenofovir base as a function of the incorporated levels of stearylamine. It is important to note that tenofovir entrapment was very low in the absence of stearylamine, due to its leakage through the lipid bilayers during hydration of the lipid phase. The obtained data show that increasing stearylamine level was accompanied by an increase in EF. For example, 39\% and $68 \%$ were the EFs for formulations $\mathrm{A}$ and $\mathrm{B}$, which were prepared with $7.5 \%$ and $15 \%(\mathrm{w} / \mathrm{w})$ stearylamine, respectively. This result could be attributed to the ionization of tenofovir into its negatively charged conjugated acid that may interact with the positive stearylamine component of the lipid bilayers. ${ }^{27,35}$ Deleers et al ${ }^{36}$ stated a rigidization of the liposome bilayers by the addition of stearylamine to the lipid pool, 
Table 3 Robustness data (mean \pm relative standard deviation) expressed as nominal, low, and high values for flow rate, injection volume, column temperature, organic-phase fraction, and $\mathrm{pH}$ value of mobile-phase variations

\begin{tabular}{|c|c|c|c|c|c|c|c|}
\hline Parameters & Change & $\begin{array}{l}\text { Retention } \\
\text { time }\end{array}$ & $\begin{array}{l}\text { Capacity } \\
\text { factor (k') }\end{array}$ & Symmetry & Area & Resolution & $\begin{array}{l}\text { Plates (half } \\
\text { width), m }\end{array}$ \\
\hline Nominal condition & & $4.055 \pm 0.092$ & $0.674 \pm 0.225$ & $0.668 \pm 0.744$ & $218.63 \pm 0.152$ & $15.748 \pm 0.497$ & $31,804 \pm 1.122$ \\
\hline \multirow[t]{2}{*}{ Flow rate, $\mathrm{mL} / \mathrm{min}$} & (Low) 0.975 & $4.149 \pm 0.158$ & $0.713 \pm 0.414$ & $0.671 \pm 0293$ & $224.4 \pm 0.293$ & $15.838 \pm 0.092$ & $32,472 \pm 0.091$ \\
\hline & (High) 1.025 & $3.966 \pm 0.065$ & $0.637 \pm 0.209$ & $0.666 \pm 0.551$ & $213.58 \pm 0.069$ & $15.652 \pm 0.269$ & $31,360 \pm 1.226$ \\
\hline \multirow[t]{2}{*}{ Injection volume, $\mu \mathrm{L}$} & (Low) 7.5 & $4.314 \pm 0.085$ & $0.780 \pm 0.170$ & $0.710 \pm 0.928$ & $161.113 \pm 0.203$ & $15.352 \pm 0.419$ & $35,8 \mathrm{II} \pm \mathrm{I} .468$ \\
\hline & (High) I2.5 & $4.326 \pm 0.14$ & $0.785 \pm 0.253$ & $0.716 \pm 0.293$ & $272.5 \pm 0.231$ & $15.166 \pm 0.318$ & $34,952 \pm 1.673$ \\
\hline \multirow[t]{2}{*}{ Temperature, ${ }^{\circ} \mathrm{C}$} & (Low) 24 & $3.897 \pm 0.462$ & $0.612 \pm 0.419$ & $0.680 \pm 0.365$ & $201.6 \pm 0.275$ & $15.173 \pm 0.397$ & $31,526 \pm 0.306$ \\
\hline & (High) 28 & $3.865 \pm 0.076$ & $0.595 \pm 0.203$ & $0.688 \pm 0.704$ & $202.733 \pm 0.138$ & $15.108 \pm 0.591$ & $32,442 \pm 1.395$ \\
\hline \multirow[t]{2}{*}{ Organic phase, \% } & (Low) 4.9 & $4.082 \pm 0.164$ & $0.685 \pm 0.430$ & $0.660 \pm 0.606$ & $224.9 \pm 0.275$ & $15.807 \pm 0.336$ & $30,072 \pm 1.328$ \\
\hline & (High) 5.1 & $3.89 \pm 0.068$ & $0.605 \pm 0.171$ & $0.655 \pm 0.648$ & $217.7 \pm 0.369$ & $15.493 \pm 0.362$ & $29,410 \pm 0.134$ \\
\hline \multirow[t]{2}{*}{$\mathrm{pH}$} & (Low) 7.3 & $3.799 \pm 0.060$ & $0.568 \pm 0.133$ & $0.632 \pm 0.527$ & $234.0 \pm 0.307$ & $14.139 \pm 0.396$ & $27,362 \pm 1.247$ \\
\hline & (High) 7.5 & $3.475 \pm 0.148$ & $0.434 \pm 0.553$ & $0.671 \pm 0.523$ & $233.8 \pm 0.349$ & $16.391 \pm 0.484$ & $28,403 \pm 1.974$ \\
\hline
\end{tabular}

Table 4 Entrapment efficiency and apparent permeability (Caco2 transwells, $n=4$ ) data for tenofovir liposomal formulations

\begin{tabular}{lll}
\hline Formulation \# & $\begin{array}{l}\text { Entrapment } \\
\text { efficiency } \mathbf{( \% )}\end{array}$ & $\begin{array}{l}\text { Apparent permeability } \\
\mathbf{( c m} / \mathbf{s})\end{array}$ \\
\hline Tenofovir solution A & - & $3.7 \mathrm{IE}-07 \pm 1.2 \mathrm{IE}-07$ \\
Formulation A & $39.8 \pm 8.09$ & $4.18 \mathrm{E}-06 \pm 1.92 \mathrm{E}-07$ \\
Formulation B & $68.1 \pm 2.55$ & $1.19 \mathrm{E}-06 \pm 1.5 \mathrm{IE}-07$
\end{tabular}

Notes: Formulations were prepared using $50 \mathrm{mg}$ cholesterol, either $7.5 \%$ (formulation A) or 15\% (formulation B) stearylamine as a positive charge imparting agent and an amount of phospholipon $100 \mathrm{H}$ to make a total lipid pool of $150 \mathrm{mg}$. Tenofovir solution A was prepared in correspondence to the EF value of Formulation $A$ in isotonic phosphate buffer.

Abbreviation: $E F$, entrapment efficiency.

and hence, higher EF was observed. The long stearyl chain inserted into the lipid bilayers could decrease its permeability to the entrapped drug. ${ }^{37}$ Having this significant discrimination between the two formulae would suggest the success of the developed analytical method to monitor the incorporation of tenofovir into this liposomal matrix.

Caco-2 human colon carcinoma cell line is often used as an in vitro screening for evaluating the rate of intestinal drug absorption..$^{28}$ The Caco-2 in vitro permeability experiment was utilized to evaluate whether tenofovir liposomal drug delivery provided enhanced drug permeation through biological membranes compared to its aqueous solution form. The application of the Caco-2 model for the assessment of liposomal-encapsulated drugs is a useful critical path tool to predict the bioavailability of complex pharmaceutical drug delivery systems. In this regard, permeability samples were diluted with mobile phase and injected into the HPLC using the described method. Representative chromatograms for Caco-2 analysis for formulations A and B (120 min time point) are shown in Figure 2E and F, respectively. The chromatograms show that there was no coelution of the drug with any of the cultural medium components. Table 4 summarizes the apparent permeability $\left(\mathrm{P}_{\text {app }}\right)$ values for the proposed two liposomal formulations. It was observed that the increased $\mathrm{EF}$ of tenofovir within the liposomal vesicles allowed a 10 -fold increase in the permeability rate when comparing the liposomal formulations versus tenofovir drug solution. In addition, increase in the incorporated stearylamine percentage within the liposomal formulations was accompanied by a significant $(p<0.05)$ decrease in the drug permeability. Krishna et al ${ }^{38}$ explained this observation by the presence of positive charge of the liposomal lamellae that could increase the drug retention by the cells rather than its permeation to the BA side. Consequently, the proposed approach demonstrated the potential of liposomal formulations to enhance the permeability of tenofovir. Further formulation screening studies are in progress for optimizing the formulation parameters for higher EF and liposomal formulation permeability using the validated analytical method.

\section{Conclusion}

The development of effective characterization tools is essential for product understanding. Herein, an efficient, yet simple, isocratic RP-HPLC method has been developed and validated according to USP category I for the pharmaceutical characterization of liposomal formulations that are targeted to enhance the bioavailability of hydrophilic HIV drugs. The method has been successfully applied for the pharmaceutics assessment of model tenofovir liposomal drug delivery formulations and the biopharmaceutical assessment of in vitro permeability. In summary, the model liposomal formulations effectively encapsulated tenofovir and were able to significantly enhance in vitro permeability. Predictively, the model liposomal formulations should protect the prodrug and enhance the oral bioavailability of tenofovir to improve clinical efficacy.

\section{Disclosure}

This scientific publication reflects the views of the authors and should not be construed to represent FDA's views or 
policies. The authors report no conflicts of interest in this work.

\section{References}

1. Sax PE, Wohl D, Yin MT et al; GS-US-292-0104/0111 Study Team. Tenofovir alafenamide versus tenofovir disoproxil fumarate, coformulated with elvitegravir, cobicistat, and emtricitabine, for initial treatment of HIV-1 infection: two randomised, double-blind, phase 3, non-inferiority trials. Lancet. 2015;385(9987):2606-2615.

2. Ray AS, Fordyce MW, Hitchcock MJ. Tenofovir alafenamide: a novel prodrug of tenofovir for the treatment of human immunodeficiency virus. Antiviral Res. 2016;125:63-70.

3. VIREAD® (tenofovir disoproxil fumarate). Viread-Labeling [package insert]. USA: Gilead Sciences, Inc. Vol 21-356-GS-035; 2015:1-50. Available from: http://www.gilead.com/ /media/files/pdfs/medicines/ liver-disease/viread/viread_pi.pdf. Accessed August 16, 2016.

4. Sentenac S, Fernandez C, Thuillier A, Lechat P, Aymard G. Sensitive determination of tenofovir in human plasma samples using reversedphase liquid chromatography. J Chromatogr B Analyt Technol Biomed Life Sci. 2003;793(2):317-324.

5. O’Neil MJ, Heckelman PE, Dobbelaar PH, Roman KJ, Kenny CM, Karaffa LS. The Merck Index: An Encyclopedia of Chemicals, Drugs, and Biologicals. 15th ed. Cambridge: Royal Society of Chemistry; 2013.

6. Fung HB, Stone EA, Piacenti FJ. Tenofovir disoproxil fumarate: a nucleotide reverse transcriptase inhibitor for the treatment of HIV infection. Clin Ther. 2002;24(10):1515-1548.

7. Sharma A, Sharma US. Liposomes in drug delivery: progress and limitations. Int J Pharm. 1997;154(2):123-140.

8. Barditch-Crovo P, Deeks SG, Collier A, et al. Phase I/II trial of the pharmacokinetics, safety, and antiretroviral activity of tenofovir disoproxil fumarate in human immunodeficiency virus-infected adults. Antimicrob Agents Chemother. 2001;45(10):2733-2739.

9. Schooley R, Ruane P, Myers R, et al. Tenofovir disoproxil fumarate (TDF) for the treatment of antiretroviral experienced patients: a 48 week analysis of a randomized, double blind, placebo-controlled study. AIDS. 2000;14:S6-S7.

10. Giacalone G, Hillaireau H, Fattal E. Improving bioavailability and biodistribution of anti-HIV chemotherapy. Eur J Pharm Sci. 2015;75:40-53.

11. Pozniak A. Tenofovir: what have over 1 million years of patient experience taught us? Int J Clin Pract. 2008;62(8):1285-1293.

12. Zidan AS, Habib MJ. Maximized mucoadhesion and skin permeation of anti-AIDS-loaded niosomal gels. J Pharm Sci. 2014;103(3):952-964.

13. Varga A, Graczer E, Chaloin L, et al. Selectivity of kinases on the activation of tenofovir, an anti-HIV agent. Eur J Pharm Sci. 2013;48(1-2):307-315.

14. Hirt D, Urien S, Ekouevi DK, et al. Population pharmacokinetics of tenofovir in HIV-1-infected pregnant women and their neonates (ANRS 12109). Clin Pharmacol Ther. 2009;85(2):182-189.

15. Sosnik A, Chiappetta DA, Carcaboso AM. Drug delivery systems in HIV pharmacotherapy: what has been done and the challenges standing ahead. J Control Release. 2009;138(1):2-15.

16. Eloy JO, Claro de Souza M, Petrilli R, Barcellos JPA, Lee RJ, Marchetti JM. Liposomes as carriers of hydrophilic small molecule drugs: strategies to enhance encapsulation and delivery. Colloids Surf B Biointerfaces. 2014;123(0):345-363.

17. Desormeaux A, Bergeron MG. Liposomes as drug delivery system: a strategic approach for the treatment of HIV infection. J Drug Target. 1998;6(1):1-15.

18. Jin SX, Bi DZ, Wang J, Wang YZ, Hu HG, Deng YH. Pharmacokinetics and tissue distribution of zidovudine in rats following intravenous administration of zidovudine myristate loaded liposomes. Pharmazie. 2005;60(11):840-843.

19. FDA-Guidance-for-Industry. Liposome Drug Products; Chemistry, Manufacturing, and Controls; Human Pharmacokinetics and Bioavailability; and Labeling Documentation. Guidance for Industry. 2015;Revision I(Draft Guidance):1-14. Available from: http://www. fda.gov/downloads/drugs/guidancecomplianceregulatoryinformation/ guidances/ucm070570.pdf. Accessed June 13, 2016.
20. Kandagal PB, Manjunatha DH, Seetharamappa J, Kalanur SS. RP-HPLC method for the determination of tenofovir in pharmaceutical formulations and spiked human plasma. Anal Lett. 2008;41(4):561-570.

21. Rodriguez-Novoa S, Morello J, Barreiro P, et al. Switch from ritonavirboosted to unboosted atazanavir guided by therapeutic drug monitoring. AIDS Res Hum Retroviruses. 2008;24(6):821-825.

22. Bezy V, Morin P, Couerbe P, Leleu G, Agrofoglio L. Simultaneous analysis of several antiretroviral nucleosides in rat-plasma by high-performance liquid chromatography with UV using acetic acid/hydroxylamine buffer - test of this new volatile medium-pH for HPLC-ESI-MS/MS. J Chromatogr B Analyt Technol Biomed Life Sci. 2005;821(2):132-143.

23. Le Saux T, Chhun S, Rey E, et al. Quantification of seven nucleoside/ nucleotide reverse transcriptase inhibitors in human plasma by highperformance liquid chromatography with tandem mass-spectrometry. J Chromatogr B Analyt Technol Biomed Life Sci. 2008;865(1-2):81-90.

24. Vourvahis M, Tappouni HL, Patterson KB, et al. The pharmacokinetics and viral activity of tenofovir in the male genital tract. J Acquir Immune Defic Syndr. 2008;47(3):329-333.

25. Anthonypillai C, Gibbs JE, Thomas SA. The distribution of the antiHIV drug, tenofovir (PMPA), into the brain, CSF and choroid plexuses. Cerebrospinal Fluid Res. 2006;3:1-1.

26. Rezk NL, Crutchley RD, Kashuba ADM. Simultaneous quantification of emtricitabine and tenofovir in human plasma using high-performance liquid chromatography after solid phase extraction. J Chromatogr B Analyt Technol Biomed Life Sci. 2005;822(1-2):201-208.

27. Zidan AS, Spinks C, Fortunak J, Habib M, Khan MA. Near-infrared investigations of novel anti-HIV tenofovir liposomes. AAPS J. 2010; 12(2):202-214.

28. Volpe DA, Faustino PJ, Ciavarella AB, et al. Classification of drug permeability with a Caco-2 cell monolayer assay. Clin Res Regul Affairs. 2007;24(1):39-47.

29. Palumbo P, Picchini U, Beck B, van Gelder J, Delbar N, DeGaetano A. A general approach to the apparent permeability index. $J$ Pharmacokinet Pharmacodyn. 2008;35(2):235-248.

30. Suhel P, Bahel US, Rajesh P, Probhakar D, Engla G, Nagar PN. Spectrophotometric method development and validation for simultaneous estimation of tenofovir disoproxil fumarate and emtricitabine in bulk drug and table dosage form. Int J Pharm Clin Res. 2009;1(1):28-30.

31. International conference on harmonisation of technical requirements for registration of pharmaceuticals for human use; 2005. ICH Harmonised Tripartite Guideline. Validation of Analytical Procedures: Text and Methodology Q2 (R1). Available from: http://www.ich.org/fileadmin/ Public_Web_Site/ICH_Products/Guidelines/Quality/Q2_R1/Step4/ Q2_R1_Guideline.pdf. Accessed January 11, 2017.

32. Ramaswamy A, Arul Gnana Dhas AS. Development and validation of analytical method for quantitation of emtricitabine, tenofovir, efavirenz based on HPLC. Arabian J Chem. Epub August 27, 2014. Available at: http://www.sciencedirect.com/science/article/pii/S1878535214001695. Accessed August 16, 2016.

33. Ashour HK, Belal TS. New simple spectrophotometric method for determination of the antiviral mixture of emtricitabine and tenofovir disoproxil fumarate. Arabian J Chem. Epub June 28, 2013. Available at: http://www.sciencedirect.com/science/article/pii/S1878535213001883. Accessed August 16, 2016.

34. CDER-Guidline. Reviewer guidance: validation of chromatographic methods. Reviewer Guidance. 1994:1-33. Available from: http://www.fda.gov/ downloads/Drugs/Guidances/UCM134409.pdf. Accessed August 27, 2015.

35. Kulkarni SB, Betageri GV, Singh M. Factors affecting microencapsulation of drugs in liposomes. J Microencapsul. 1995;12(3):229-246.

36. Deleers M, Guilmin T, Vandenbranden M, Ruysschaert JM. Thermotropic properties of dipalmitoyl phosphatidyl choline stearylamine liposomes. Pharmacol Res Commun. 1982;14(4):333-339.

37. Deleers M, Malaisse WJ. Ionophore-mediated calcium exchange diffusion in liposomes. Biochem Biophys Res Commun. 1980;95(2): 650-657.

38. Krishna G, Chen K, Lin C, Nomeir AA. Permeability of lipophilic compounds in drug discovery using in-vitro human absorption model, Caco-2. Int J Pharm. 2001;222(3):77-89. 
Clinical Pharmacology: Advances and Applications is an international, peer-reviewed, open access journal publishing original research, reports, reviews and commentaries on all areas of drug experience in humans. The manuscript management system is completely online and includes a very quick and fair peer-review system, which is all easy to use.

Submit your manuscript here: https://www.dovepress.com/clinical-pharmacology-advances-and-applications-journal
Visit http://www.dovepress.com/testimonials.php to read real quotes from published authors. 